\title{
Dynamic response of boundary-layer turbulence to oscillatory shear
}

\author{
G. J. Brereton \\ Department of Mechanical Engineering and Applied Mechanics, The University of Michigan, \\ Ann Arbor, Michigan 48109 \\ W. C. Reynolds \\ Department of Mechanical Engineering, Stanford University, Stanford, California 94305
}

(Received 23 January 1989; accepted 6 September 1990)

\begin{abstract}
The temporal response of a well-developed turbulent boundary layer to the superposition of oscillatory shear has been measured experimentally, over a wide range of frequencies. The response is primarily a periodic organization in magnitude of components of the turbulent velocity field at the forcing frequency. Oscillatory production of turbulence arises predominantly as a modulation of the mean production process in the parent boundary layer. Close to the wall, the relative phases of response of components of turbulent kinetic energy indicate that temporal redistribution of turbulent kinetic energy is driven by robust coherent motions of the underlying mean flow. The local directions of redistribution deduced from these measurcments indicate a wall impingement (splatting) effect, consistent with characterizations from numerical simulation.
\end{abstract}

\section{INTRODUCTION}

In efforts to improve understanding and prediction of turbulent flows, categorization of these flows according to the kinds of deformation they undergo has proved a rewarding exercise both for consolidating present understanding and for focusing on the kinds of experiments needed to supply further insight. For example, in the case of homogeneous turbulent flows, ${ }^{\prime}$ a variety of different experimental results-on isotropic turbulence, turbulence under rotation, turbulence returning to isotropy, undergoing plane strain, and undergoing uniform shear--are available as target data for turbulence modelers, and as a point of departure for further studies into the effects of strain and rotation on homogeneous turbulence. In the case of more complex turbulent flows such as boundary layers, decades of research have resulted in a large literature on the consequences of steady deformation resulting from pressure gradients, buoyancy, curvature, Coriolis forces, etc., and on the prediction of their effects. However, relatively little is known about the interactions between time-dependent deformation and turbulence in boundary layers. In particular, the effects of time-dependent shear are not well understood; their importance in turbomachinery and in aerodynamic applications provides motivation for further study.

For reasons of experimental and analytical expedience, nearly all studies of time-dependent deformation of boundary layers have involved oscillating, sinusoidal variations in fluid velocity. The pioneering work of Karlsson ${ }^{2}$ on the effects of sinusoidal variations in free-stream velocity upon a boundary layer with no mean pressure gradient established the comparability of many time-averaged measures in unsteady flow and those same measures in equivalent steady flows; their invariance to different frequcncies of free-stream unsteadiness was also noted. These findings were generally confirmed by later studies and were found to hold for unsteady flow in diffusers, channels, and pipes..$^{3-6}$ However, insight into the detailed temporal structure of turbulence under unsteady conditions has been limited since simultaneous measurements of three components of the velocity field have yet to be made, and two-component measurements only appear to have been mastered in a few studies, ${ }^{3,7,8}$

Recent computations of unsteady turbulent wallbounded flows have highlighted the shortage of detailed measurements of the time-dependent response of boundarylayer turbulence to organized unsteadiness. Reynolds-stress closures, ${ }^{9} k-\epsilon$ models ${ }^{9}$ and pseudoenergy/pseudovorticity models ${ }^{10}$ have all been applied to the case of pulsating turbulent pipe flow with reasonable success. However, the kinds of experimental data available for this flow provide only limited guidance as to the specific strengths and weaknesses of unsteady turbulence models, or to where improvement is needed. Computational predictions have also been performed for unsteady turbulent flow over flat plates and in channels. Typically, steady-flow closures have been extended for application to flows characterized by oscillation about their mean condition. The sophistication of these closure ranges from pioneering eddy-viscosity models ${ }^{11}$ to more recent multiscale representations ${ }^{12}$ of turbulence in unsteady boundary layers. However, evaluation of the performance of these models continues to be restricted by a shortage of detailed experimental data, and a limited understanding of temporal behavior of turbulent flow undergoing forced transients.

The study reported in this paper was undertaken with a view to improving understanding of both the mean and the temporal nature of unsteady turbulent wall-bounded fiow. Well-resolved simultaneous measurements of the $u$ and $v$ components of the velocity field were made within a boundary layer, the free stream beyond which underwent sinusoidal oscillations in velocity about its mean, over a wide range of frequencies. The mean condition of the turbulent bound- 
ary layer was found to be insensitive to sinusoidal oscillation in free-stream velocity at any of the frequencies studied. Time-averaged profiles of velocities and of components of the Reynolds-stress tensor scarcely differed from those in steady flow at the mean conditions, attesting to the robustness of the turbulent motions of the boundary layer to oscillatory shear. These results are reported elsewhere ${ }^{13}$ Insights into the dynamic response of boundary-layer turbulence to forced unsteady shear, based on these measurements, are presented in this paper.

\section{DECOMPOSITION, AVERAGING, AND TRANSPORT EQUATIONS}

When describing time-dependent flows in which the velocity and pressure fields respond to a perturbation of known form, it is useful to express dependent variables in the equations of motion via a triple decomposition, as proposed by Hussain and Reynolds. ${ }^{14}$ Thus an instantaneous measure of a turbulent quantity $f(\mathbf{x}, t)$ may be described as the summed contribution of three parts:

$$
f(\mathbf{x}, t)=\bar{f}(\mathbf{x})+\ddot{f}(\mathbf{x}, t)+f^{\prime}(\mathbf{x}, t) .
$$

These components are the mean or time-averaged one, the deterministic, periodic, or oscillatory one, and the turbulent component, respectively. The overbar and prime indicate time-averaged and turbulent measures, respectively, whereas the tilde (for which $\sim$ replaces $\sim$ for longer arguments), denotes the periodic or oscillatory component of the quantity. In order to separate any variable into these three components, two averaging procedures are required. One possible combination is (i) the phase average or ensemble average of the quantity, $\langle f(\mathbf{x}, t)\rangle$, defined as

$$
\langle f(\mathbf{x}, t)\rangle=\bar{f}(\mathbf{x})+\tilde{f}(\mathbf{x}, t)=\lim _{N \rightarrow \infty} \frac{1}{N} \sum_{n=0}^{N-1} f(\mathbf{x}, t+n \tau),
$$

where $\tau$ is the period of the cycle; and (ii) the time average,

$$
\bar{f}(\mathbf{x})=\lim _{N \rightarrow \infty} \frac{1}{N} \sum_{n=0}^{N-1} f\left(\mathbf{x}, t_{0}+n \Delta t\right)
$$

where

\section{$N \Delta t \gg \tau$.}

When the periodic nature of the flow allows successive cycles to be considered as independent events, the phase average (i.e., the average over a large ensemble of points, each measured at the same phase with respect to a reference oscillator) effectively filters out background turbulence, while the time average removes both background turbulence and periodic contributions. Using this decomposition and these averaging procedures, equations of fluid motion may be devised and measurements of pertinent quantities made for each of the mean, periodic, and turbulent fields of flow.

Of particular interest to this study is the equation describing transport of the Reynolds stress within the periodic field of flow, i.e., the component of $u_{i}^{\prime} u_{j}^{\prime}$, or for the sake of brevity $r_{i j}$, which oscillates about its mean value in response to periodic motion induced within the boundary layer. This transport equation for the oscillatory component of $r_{i j}\left(\tilde{r}_{i j}\right.$ or $\left\langle r_{i j}\right\rangle-\bar{r}_{i j}$ ) may be deduced from the phase-averaged Reynolds-stress budget:

$$
\frac{D}{D t}\left\langle r_{i j}\right\rangle=\left\langle P_{i j}\right\rangle+\left\langle T_{i j}\right\rangle-\left\langle D_{i j}\right\rangle-\left\langle J_{i j k}\right\rangle_{, k}
$$

in which the substantial derivative follows the phase-averaged unsteady motion in the form

$$
\frac{D}{D t}\langle\rangle=\frac{\partial}{\partial t}\langle\rangle+\left\langle u_{j}\right\rangle \frac{\partial}{\partial x_{j}}\langle\rangle \text {. }
$$

The production, pressure strain, dissipation, and diffusive flux terms $\left(\left\langle P_{i j}\right\rangle,\left\langle T_{i j}\right\rangle,\left\langle D_{i j}\right\rangle\right.$, and $\left.\left\langle J_{i j k}\right\rangle\right)$ of (1) are then the phase-averaged equivalents of their steady-flow counterparts. Subtraction of the time-averaged form of (1) allows the oscillatory Reynolds-stress budget to be written in a form analogous to its counterpart in steady turbulent flow, name$1 y$,

$$
\frac{D}{D t}\left(\tilde{r}_{i j}\right)=\widetilde{P}_{i j}+\widetilde{T}_{i j}-\widetilde{D}_{i j}-\widetilde{J}_{i j k, k} .
$$

The substantial derivative follows the organized unsteady motion, such that

$$
\frac{D}{D t}(\tilde{)})=\frac{\partial}{\partial t}(\tilde{)})+U_{j} \frac{\partial}{\partial x_{j}}(\tilde{)})+\tilde{u}_{j} \frac{\widetilde{\partial}}{\partial x_{j}(\tilde{\tau})}
$$

and the oscillatory production-rate tensor $\widetilde{P}_{i j}$ is

$$
\widetilde{P}_{i j}=-\left(r_{i k} \frac{\widetilde{\partial \bar{u}_{j}}}{\partial x_{k}}+r_{j k} \frac{\widetilde{\partial \bar{u}_{i}}}{\partial x_{k}}\right)-\left(\tilde{r}_{i k} \frac{\partial U_{j}}{\partial x_{k}}+\tilde{r}_{j k} \frac{\partial U_{i}}{\partial x_{k}}\right) \text {. }
$$

The tensors describing the rates of oscillatory pressure strain, dissipation, and diffusive flux $\left(\widetilde{T}_{i j}, \widetilde{D}_{i j}\right.$, and $\left.\widetilde{J}_{i j k}\right)$ are then

$$
(2 / \rho) \widetilde{p^{\prime} s_{i j}}, \quad 2 \widetilde{v u_{i, k}^{\prime} u_{j, k}^{\prime}}
$$

and

$$
\widetilde{u_{i}^{\prime} u_{j}^{\prime} u_{k}^{\prime}}+(1 / \rho)\left(\widetilde{p^{\prime} u_{i}^{\prime}} \delta_{j k}+\widetilde{p^{\prime} u_{j}^{\prime}} \delta_{i k}\right)-v \tilde{r}_{i, k}
$$

where $s_{i j}$ is the turbulent component of the strain-rate tensor and $\delta_{i j}$ is the Kronecker delta. Equation (3) may be thought of as describing the behavior of the relatively unorganized turbulence within the organized oscillatory field of flow.

In the experiments of this study, ${ }^{13}$ profiles of the oscillatory turbulence quantities, $\widetilde{u^{\prime} u^{\prime}}, \widetilde{v^{\prime} v^{\prime}}$, and $\widetilde{u^{\prime} v^{\prime}}$, were measured together with the mean and periodic velocities: $U, V, \tilde{u}$, and $\tilde{v}$. These measurements were made for oscillatory shear over a range of frequencies for which quasisteady flow was approached at one extreme, and slug-like Stokes flow at the other. The highest frequencies were smaller than estimates of the bursting frequency by factors of about 2 . The experiments covered a range of Strouhal numbers $\left(\omega \delta / U_{\infty}\right)$ from 0.06 to 1.2 with a corresponding range of Reynolds number based on Stokes-layer thickness $\left(\mathrm{Re}_{l}\right.$, where $l=\sqrt{2 v / \omega}$ ) from 1100 to 240 . They enabled time-dependent evolutions of three components of the $\tilde{r}_{i j}$ tensor to be studied, together with the dominant terms of their production-rate tensors.

\section{OVERVIEW OF EXPERIMENTS AND MEASUREMENT TECHNIQUES}

In the water-tunnel experiments of this study (Fig. 1), a sinusoidal motion of prescribed amplitude was superim- 


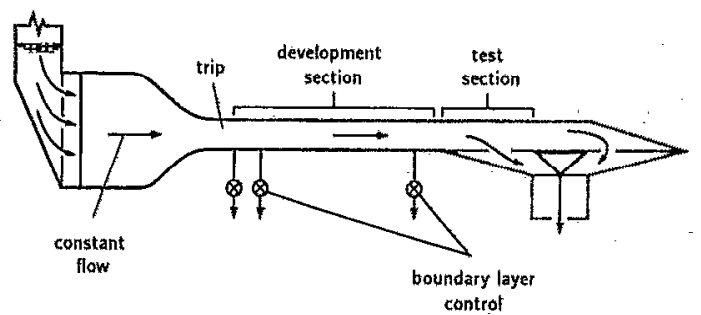

FIG. 1. Closed-loop water tunnel.

posed on an otherwise steady mainstream, beyond a twodimensional (mean) turbulent boundary layer on a smooth flat wall. This unsteady motion was induced in the test section of the apparatus by dynamically controlled suction of free-stream fluid at the tunnel wall opposite and far away from the edge of the test boundary layer. Total drainage of free-stream fiuid was maintained at a constant volume-flow rate, while the local distribution of fluid removal from the test section was controlled dynamically. In this way, effects of free-stream unsteadiness could be imposed downwind of a boundary layer that had developed under nominally steady constant-pressure upstream conditions, thereby avoiding complications that might arise from boundary-layer initiation and laminar-turbulent transition under unsteady conditions. The apparatus thus enabled unsteadiness to be induced downstream of the development section of the apparatus, at the exit of which the boundary layer was characterized by a Reynolds number $\left(\operatorname{Re}_{\theta}\right)$ of 3100 , having developed with a free-stream velocity of $0.74 \mathrm{~m} / \mathrm{sec}$.

At the station at which detailed measurements were made, the mean condition of the local pressure gradient was adverse, with the Clauser parameter $\beta$ taking a value of 6.3 . A periodic motion was induced in the free stream, of the form $\tilde{u}_{\infty}=A \cos \omega t$, where $\omega$ is the circular frequency of oscillation. The combined effect of superposition of an oscillatory flow upon a steady one was to create a time-dependent free-stream velocity $\left\langle u_{\infty}\right\rangle$ (the phase-averaged free-stream velocity, equal to $\left.U_{\infty}+\tilde{u}_{\infty}\right)$. Oscillations were induced at seven different frequencies from 0.1 to $2.0 \mathrm{~Hz}$. In this turbulent boundary layer, these periods corresponded to approximately 3500 and 175 viscous time scales, respectively. For forced oscillation at each of these frequencies, the amplitude of the free-stream velocity $(A)$ was held constant, at $15 \%$ of $U_{\infty}$. Local values of time-averaged quantities such as $U, V, \overline{u^{\prime} u^{\prime}}, \overline{v^{\prime} v^{\prime}}$, and $-\overline{u^{\prime} v^{\prime}}$ were practically invariant to different frequencies of forced unsteadiness, as were $u_{\tau}$ and $\delta$. Though not always identical, these measures exhibited only slight departures from their counterparts in steady flow at the same mean external-flow conditions. ${ }^{13}$

Simultaneous measurements of the $u$ and $v$ components of the velocity field were made within the boundary layer on the top wall of the test section, with a two-color laserDoppler anemometer. At the measurement station for which results are reported in this study, the approximate dimensions of the measuring volume corresponded to 8.3 viscous units in length and 2.5 viscous units in diameter. The temporal response of the velocity field was resolved through conditioning samples of these measurements on the phase of

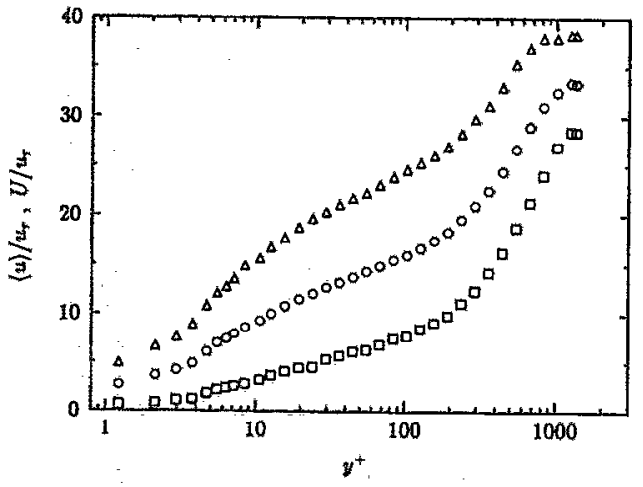

FIG. 2. Boundary-layer profiles of $U$ and $\langle u\rangle$ at $0.2 \mathrm{~Hz} ; O, U / u_{r} ; \Delta$, $\langle u\rangle / u_{r} ; \square,\langle u\rangle / u_{r}$. Profiles of $\langle u\rangle$ are conditioned on the phase of maximum and minimum $\left\langle u_{\infty}\right)$ and normalized by the mean friction velocity.

the oscillating mechanism that controlled suction of freestream fluid (and thus determined the free-stream velocity). Phase averages were made over 500 cycles, and at 512 discrete evenly spaced times within each cycle.

\section{FORCING CONDITIONS}

In the experiments of this study, oscillatory shear was induced through superposition of a sinusoidally varying velocity upon an otherwise steady free stream-a forced, local, time-dependent boundary condition for the flow. For each of the seven frequencies at which oscillatory shear was induced, $\tilde{u}_{\infty}$ was almost purely sinusoidal, its fundamental frequency accounting for about $99 \%$ of the total harmonic content. The effect of this induced shear upon the boundarylayer flow is illustrated in Figs. 2 and 3, in which profiles of the mean streamwise velocity and rms levels of streamwise turbulence are shown, together with ensemble-averaged profiles of these measures at the phases corresponding to the extremes in free-stream velocity. These data are for oscillation at $0.2 \mathrm{~Hz}$, a frequency at which the behavior of the boundary layer is similar to, though certainly not identical to, its quasisteady form. Each profile in these figures is normalized by the friction velocity deduced from the mean ve-

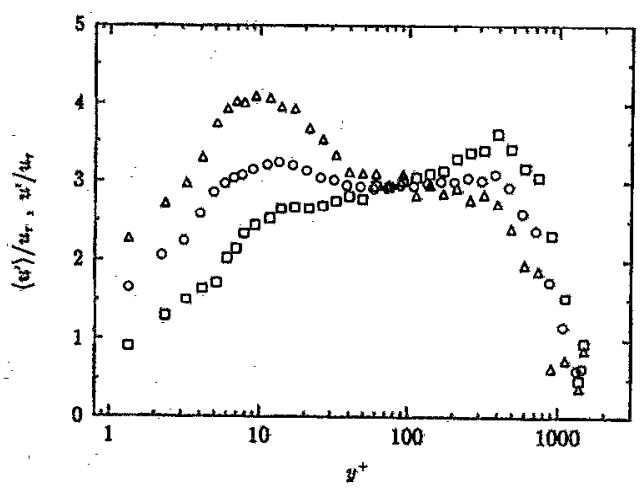

FIG. 3. Boundary-layer profiles of $\left\langle u^{\prime}\right\rangle$ and $u^{\prime}$ at $0.2 \mathrm{~Hz} ; O, u^{\prime} / u_{r} ; \Delta$, $\left\langle u^{\prime}\right\rangle / u_{r} ; \square,\left\langle u^{\prime}\right\rangle / u_{r}$. Profiles of $\left\langle u^{\prime}\right\rangle$ correspond to maximum and minimum $\left\langle u_{*}\right\rangle$, respectively, and are normalized by the mean friction velocity. 
locity profile. Thus the deviations of phase-averaged profiles from their mean are indicative of the local amplitude of variation in $\langle u\rangle$ and $\left\langle u^{\prime}\right\rangle$. It is clear from these figures that the free-stream oscillation exerts a strong influence upon the time-dependent velocity and turbulence fields, which is evident throughout the boundary layer.

The utility of a phase-averaged representation becomes apparent when ensemble-averaged measures of streamwise velocity (shown at two of the 512 phases in Fig. 2) are differentiated in the wall-normal direction. The differentiation procedure employed was to make four-point piecewise parabolic least-squares fits along the $\langle u\rangle$ profile and evaluate the gradient from piecewise-cubic splines through the fits to the data. The small degree of smoothing inherent to this method was necessitated by the usual noise problems, which arose when direct differences were used to evaluate gradients from discrete data. The oscillatory component of velocity gradient could then be extracted and was expressed as $\partial \tilde{u} / \partial y_{1}$, the Fourier amplitude of $\partial \tilde{u} / \partial y$ at the forcing frequency. Since the harmonic content at the fundamental frequency exceeded $97 \%$ of the total harmonic content of $\tilde{u}$ throughout the boundary layer, at each forcing frequency, $\partial \tilde{u} / \partial y$ could be described quite adequately by $\partial \hat{u} / \partial y_{1}$ and an accompanying phase measure. The magnitude of the other oscillatory component of shear in this flow, $\partial \tilde{v} / \partial x$, could always be assumed small in comparison and so $\widehat{\partial u} / \partial y_{1}$ was taken as the amplitude of oscillatory shear.

Boundary-layer profiles of the amplitude of oscillatory shear, the forcing function under consideration, are shown in Fig. 4 for all frequencies of unsteadiness considered in this experiment. All profiles are of similar shape and those measured at high frequencies $(1.6 \mathrm{~Hz}, 2.0 \mathrm{~Hz})$ are in good qualitative agreement with the high-frequency asymptote for oscillatory shear, described by the quasilaminar analytical solution to the oscillatory $x$-momentum equation:

$$
\frac{\partial \tilde{u}}{\partial t}=-\frac{1}{\rho} \frac{\partial \tilde{p}}{\partial x}+v \frac{\partial^{2} \tilde{u}}{\partial y^{2}} .
$$

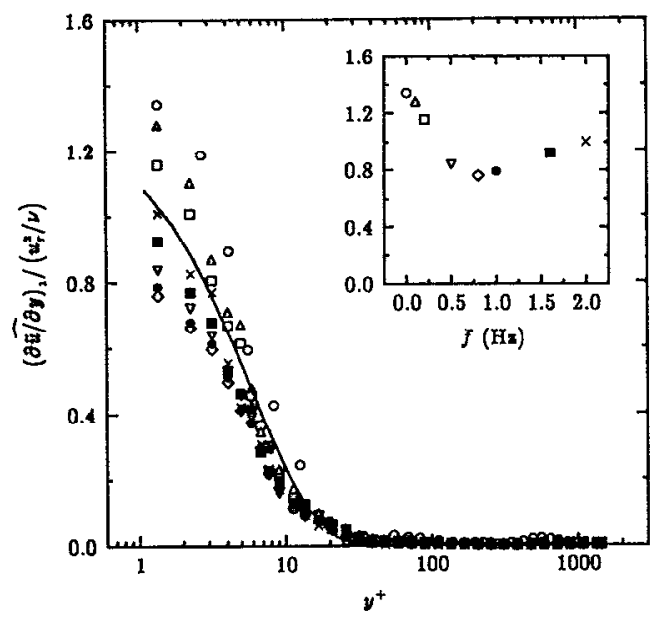

FIG. 4. Boundary-layer profiles of normalized oscillatory shear amplitude; - Stokes' solution for oscillatory shear at $2.0 \mathrm{~Hz} ; \times, 2.0 \mathrm{~Hz} ; \mathbf{0}$, $1.6 \mathrm{~Hz} ; 1.0 \mathrm{~Hz} ; 0,0.8 \mathrm{~Hz} ; \nabla, 0.5 \mathrm{~Hz} ; \square, 0.2 \mathrm{~Hz} ; \Delta, 0.1 \mathrm{~Hz} ; 0$, quasisteady. Normalization is in each case with respect to the mean friction velocity.
The solution to this equation is plotted for oscillation at 2.0 $\mathrm{Hz}$. For the case of a stationary wall and an oscillatory free stream, it may be expressed in the form

$$
\frac{\widehat{\partial \tilde{u}}}{\partial y_{1}}=\frac{\hat{u}_{1, \infty}}{\sqrt{v / \omega}} e^{-y / \sqrt{2} \hat{v} / \omega},
$$

where $\hat{u}_{1, \infty}$ is the Fourier amplitude of the free-stream velocity at its first harmonic. A quasisteady profile for oscillatory shear in this boundary layer is also included in Fig. 4. This profile was constructed from a series of steady velocity profiles under different free-stream conditions and describes the low-frequency asymptote for oscillatory shear in this flow. The ordinate of Fig. 4 is normalized with respect to the mean friction velocity $u_{\tau}$ rather than the frequency-dependent length scale, $\sqrt{v / \omega}$. Thus local magnitudes of oscillatory shear at different frequencies may be compared readily.

It may be seen from Fig. 4 that the effect of the turbulent boundary layer is to restrict the region over which oscillatory shear is most significant to $y^{+} \leqslant 30$ in this experiment. The restriction of oscillatory shear to this thin wall layer is attributed to the effectiveness of diffusive actions of turbulent motions of the boundary layer, beyond the viscous sublayer. The relative amplitudes of $\partial \tilde{u} / \partial y$ at each frequency are shown in the inset graph, at the data point closest to the wall. The same qualitative variation of shear amplitude with frequency is observed at other locations within the viscous sublayer $\left(y^{+} \leqslant 7\right)$. The well-defined curve described by data in the inset graph reflects the orderly variation with frequency between asymptotic modes of fluid behavior, dictated by the competing effects of fluid inertia and the forcing pressure gradient, which dominate flow adjacent to the wall at low and high frequencies, respectively.

It is evident from Fig. 4 that between $y^{+} \simeq 6$ and $y^{+} \simeq 30$, where interactions between oscillatory shear and the turbulence producing motions of the boundary layer are likely to be most significant, both the amplitude of oscillatory shear and its wall-normal distribution are quite similar at all frequencies in the range of this study. The experiment therefore constitutes a very interesting test case for evaluating the response of boundary-layer turbulence to different frequencies of oscillatory shear, of roughly the same amplitude at any given wall-normal position beyond the viscous sublayer.

\section{SPECTRAL RESPONSE}

Measurements of $u$ and $v$ were recorded as time series of data at evenly spaced intervals, when the free stream was steady and when it underwent forced oscillation. The period of the forced oscillation was prescribed, and so phase- and time-averaging techniques could be employed to remove mean and periodic components of velocity from data records. Thus time series of $\tilde{u}+u^{\prime}$, and $\tilde{u}$ were constructed for the forced unsteady flow. Estimates of the power spectral density distribution were made using a standard autoregressive model. ${ }^{15}$ This method proved to be generally superior to FFT estimates for analysis of time-series data in steady flow, and gave clearer descriptions of sharp spectral features in flow undergoing forced transients. All spectral data reported in this paper are for time serics of 16384 data sampled at 250 


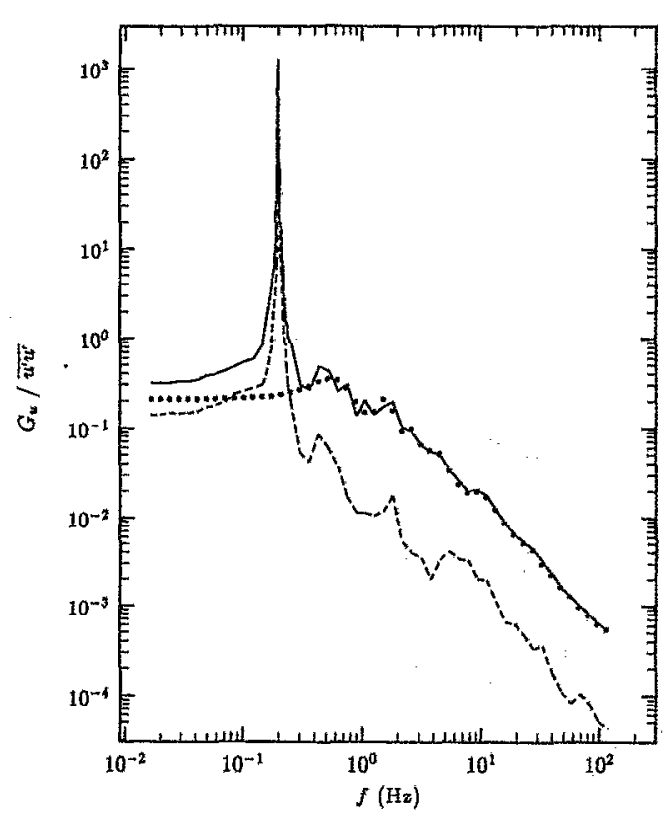

FIG. 5. Power spectral density estimations for the $\vec{u}+u^{\prime}$ time series and its components;,$- \bar{u}+u^{\prime}$ time series; $\cdots, u^{\prime}$ component of the time series; ,$-- \tilde{u}$ component of the time series.

$\mathrm{Hz}$. In the autoregressive model used for these time series, the power spectral density was described by a distribution of 600 poles. A representation of this order corresponded to the number of data in half a period, ${ }^{15}$ and was found to be an acceptable compromise between the need to provide adequate descriptions of sharp spectral peaks and the desire to avoid adding excessive amounts of noise in other parts of the spectrum.

Estimates of the power spectral density distributions deduced by this technique are shown in Fig. 5 . The data in this figure describe (i) the power spectrum of the $\tilde{u}+u^{\prime}$ time series in unsteady flow undergoing oscillatory shear at 0.2 $\mathrm{Hz}$; (ii) the power spectrum of the $\tilde{u}$ component of the time series of (i); and (iii) the power spectrum of the $u^{\prime}$ component of the time series of (i). It is predictable that the spectra for the $\tilde{u}+u^{\prime}$ and $\tilde{u}$ time series are characterized by sharp peaks at the forcing frequency of oscillation. For ease of comparison, all spectra have been normalized by $\overline{u^{\prime} u^{\prime}}$. Thus the area beneath the plotted spectral density distributions integrate to unity for case (iii), while exceeding unity in case (i).

The decomposition of this time series into its $\tilde{u}$ and $u^{\prime}$ components and their subsequent spectral estimation allowed the component contributions to the power spectrum of the turbulent flow to be examined. It may be seen from Fig. 5 that the peak in the power spectrum of the $\tilde{u}+u^{\prime}$ time series is almost entirely due to the organized oscillatory motion of $\tilde{u}$, with no detectable peak at this frequency in the turbulent component of the flow (represented by the $u^{\prime}$ spectrum). Thus there appears to be no harmonic interaction between the organized oscillatory motions and the underlying turbulent motions that contribute to $\overline{u^{\prime} u^{\prime}}$ in this boundary layer. It is also noteworthy that the inertial subrange in unsteady flow descends with a $-\frac{5}{3}$ gradient, consistent with the form of its steady-flow counterpart. The same general features were observed in the complementary power spectra and cross-spectra for $v$ and $u v^{16}$ and at other frequencies of oscillation and other locations within the boundary layer. These observations, and others, reported elsewhere, ${ }^{13}$ conform with the view that the mean behavior of turbulent motions of the boundary layer is scarcely affected by the superposition of oscillatory shear, over a wide range of frequencies.

The dominant peak in the spectrum of the $\tilde{u}+u^{\prime}$ time series is almost identical in shape to that in the $\tilde{u}$ spectrum and so may be attributed solely to the organized oscillatory motions. The small degree of broadening about the peak is then due to organized oscillatory motions and accordingly may be ascribed to minor cycle-to-cycle variations in the forcing perturbation and to limitations of the spectral estimation method. The spectral content of $\tilde{u}$ takes a very similar shape to that of $u^{\prime}$ at the higher frequencies of the estimate, indicative of imperfect decomposition of the velocity time series into $U, \tilde{u}$, and $u^{\prime}$. However, its magnitude is an order smaller and this spectral characteristic reflects the practical limitations on ideal separation of a times series into phase-averaged and random components-it is attributed to the finite number of ensembles of unsteady events within the time series.

While the power-spectral estimates shown in Fig. 5 indicate the relative contributions of different scales of motion to the long-time averages $\overline{u^{\prime} u^{\prime}}$ and $\overline{\tilde{u} \tilde{u}}$, they provide no information about the temporal frequency content of velocity measures during transients. Temporal power spectral information was deduced by applying the autoregressive spectral estimation method to blocks of data from the $u^{\prime}$ time series, chosen to correspond to specific ranges in phase of the forcing oscillatory motion. If these periods of the $u^{\prime}$ time series are assumed to be of locally stationary form, the same spectral estimation technique may be used to compare the longtime-average power spectrum to power spectra deduced over selected ranges in the phase of $\tilde{u}$.

Phase ranges were chosen as the four phase quadrants of the local velocity field $\tilde{u}=\hat{u}_{1} \cos \omega t$ (where $\hat{u}_{1}$ is the Fourier amplitude at the fundamental frequency) centered around $\omega t=0, \pi / 2, \pi$, and $3 \pi / 2$. Estimates of the spectral content of $u^{\prime}$ during each of the four quadrants and for the long-time average are shown in Fig. 6; normalization is by the long-time average $\overline{u^{\prime} u^{\prime}}$ for each spectrum. In the highfrequency region for which the estimate was most accurate, it may be seen that the scales of motion are weakly amplified, relative to the long-time estimate, during negative values of $\tilde{u}$ (the quadrant centered around $\omega t=\pi$ ) while slightly attenuated during positive excursions in $\tilde{u}$ (the quadrant centered around $\omega t=0$ ). At the particular location at which this time series in $u$ was recorded, one of the more pronounced effects of superposition of $\tilde{u}$ (and the associated oscillatory shear) was deformation of turbulent motions through growth (negative $\tilde{u}$ and reduced $\left\langle u_{\infty}\right\rangle$ ) and recession (positive $\tilde{u}$ and increased $\left.\left\langle u_{\infty}\right\rangle\right)$ of the boundary layer. Measures of $\left\langle u^{\prime} u^{\prime}\right\rangle$ at $y^{+}=400$ (shown at its rms level in Fig. 3) also illustrate that positive $\tilde{u}$ corresponds to attenuation in $u^{\prime} u^{\prime}$, and vice versa at this location in the boundary layer, tending to con- 


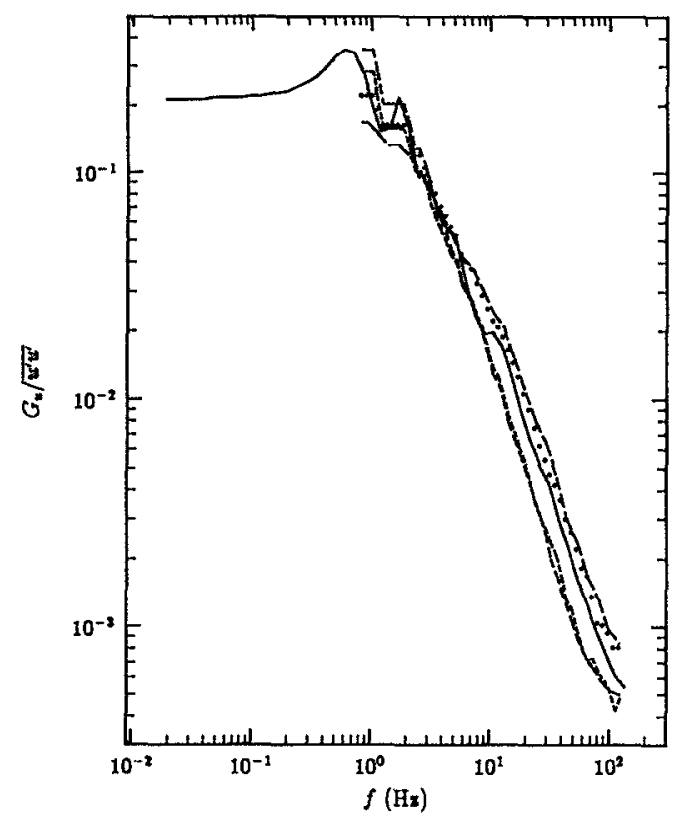

FIG. 6. Power spectral density estimates for the $u^{\prime}$ component of the time series, when conditioned upon ---, the quadrant centered around $\omega t=0 ;-$, , the quadrant centered around $\omega t=\pi / 2 ; \cdots$, the quadrant centered around $\omega t=\pi ;---$, the quadrant centered around $\omega t=3 \pi / 2 ;-$, without temporal conditioning (representative of $\left.\overline{u^{\prime} u^{\prime}}\right)$.

firm that this result is not due to uncertainty in the estimation procedure. Therefore temporal dependence of the spectral response of the streamwise component of boundary-layer turbulence to oscillatory shear is apparent at the highest frequencies of the spectral estimate-within the inertial subrange-so the response is at least weakly broadband in a temporal sense.

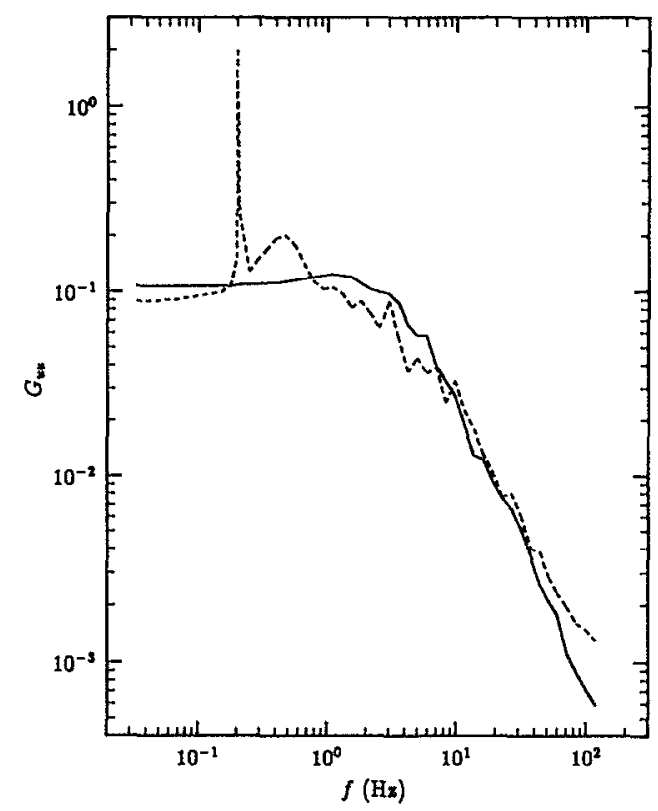

FIG. 7. Power spectral density estimates for the time series of the square of $u^{\prime}(t)$;---, unsteady flow at $0.2 \mathrm{~Hz} ;-[$ _ measure. Normalization is by the area beneath the steady spectrum.
The temporal nature of the turbulence field may also be illustrated by examining the power-spectral estimate of the square of each element of the time series for $u^{\prime}$. This spectrum is the Fourier transform of the autocorrelation function of $u^{\prime}(t) u^{\prime}(t)$, which describes periodic organization of the square (and therefore the absolute magnitude) of the turbulent component of velocity in response to the forcing perturbation. Power spectra of $u^{\prime}(t) u^{\prime}(t)$ are shown in Fig. 7 for the cases of steady and forced unsteady flow. In the case of unsteady flow, the sharp peak at $0.2 \mathrm{~Hz}$ attests to the periodic organization of $u^{\prime} u^{\prime}$ by the forcing velocity field. It is of particular significance that this peak is at least an order of magnitude greater than any other part of the spectrum. The square of the turbulent component of velocity may then be treated with some justification as a periodic quantity oscillating predominantly at the forcing frequency, for which the phase-averaging procedure conditioned on the $\tilde{u}$ field (described in Sec. II) is appropriate. The accompanying assumption that successive cycles may be treated as independent events is also lent credibility by this result-in many previous studies it has been used with no apparent justification. Comparable findings were also made for spectra of $v^{\prime}(t) v^{\prime}(t)$ and $u^{\prime}(t) v^{\prime}(t)$, at other frequencies of this study, and at other positions within the boundary layer. Therefore temporal measures of components of velocity and of double correlations of turbulent quantities could be estimated justifiably as their Fourier phases and amplitudes when phase averaged at the forcing frequency-this representation is used extensively in the following sections.

In summary, spectral estimations indicate that the timeaveraged response of boundary-layer turbulence to oscillatory shear displays no harmonic features, and retains the spectral characteristics of a steady turbulent flow. There is a strong dynamic response that is primarily a periodic organization in the absolute magnitude of turbulence, at the forcing frequency. However, it also has less pronounced broadband characteristics of a temporal nature, which are evident in parts of the turbulence spectrum as distant from the forcing frequency as within the inertial subrange.

\section{TURBULENCE PRODUCTION}

An important indicator of turbulent activity in an oscillatory field of flow is its rate of production tensor $\widetilde{P}_{i j}$. Just as its time-averaged counterpart $\bar{P}_{u^{\prime} u^{\prime}}$ is the largest contributor to production of turbulent kinetic energy in a steady turbulent boundary layer, $\widetilde{P}_{u^{\prime} u^{\prime}}$ accounts for the greatest amplitude of production of turbulent kinetic energy when oscillatory shear is superimposed on the flow. The streamwise component of turbulent kinetic energy $\widetilde{u^{\prime} u^{\prime}}$ thereby profits most from production in this oscillatory field of flow. When Eq. (3) is devised for each individual component of $\tilde{r}_{i j}$, two major terms contributing to production of $\tilde{u}^{\prime} u^{\prime}$ may be identified through order of magnitude reasoning and they are $-2 \widetilde{u^{\prime} v^{\prime}} \partial U / \partial y$ and $-2 \overline{u^{\prime} v^{\prime}} \partial \tilde{u} / \partial y$. The presence of two key terms is only an artifact of the additional stage of decomposition introduced in Sec. II, and their sum still corresponds to the oscillatory part of $\left\langle-2 u^{\prime} v^{\prime} \partial\langle u\rangle / \partial y\right\rangle$. However, this representation allows the different interactions 


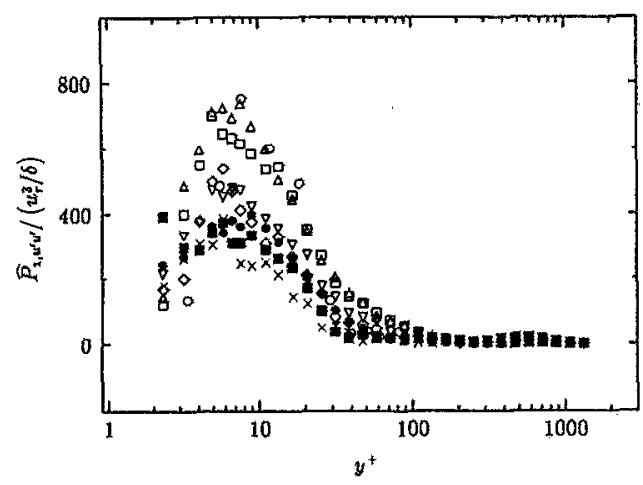

FIG. 8. Boundary-layer profiles of the amplitude of production of $\left\langle u^{\prime} u^{\prime}\right\rangle$ at its first harmonic; $X, 2.0 \mathrm{~Hz} ; \mathbf{m}, 1.6 \mathrm{~Hz} ;, 1.0 \mathrm{~Hz} ; \Delta, 0.8 \mathrm{~Hz} ; \nabla, 0.5 \mathrm{~Hz} ; \square$, $0.2 \mathrm{II} z ; \Delta, 0.1 \mathrm{Iz} ; \mathrm{O}$, quasisteady.

between the mean and oscillatory fields of flow to be identified clearly. These two production terms were always most energetic at the forcing frequency and measurements were made of their first-harmonic Fourier amplitudes and the amplitude of their sum.

Profiles of the amplitude of $\widetilde{P}_{u^{\prime} u^{\prime}}$, the net production term for $\widetilde{u}^{\prime} u^{\prime}$, are shown in Fig. 8. The ordinate normalization is by $u_{\tau}^{3} / \delta$ and wall units (also referenced to the mean friction velocity) are chosen for the abscissa. The major term accounting for production of $\overline{u^{\prime} u^{\prime}}$ (denoted $\bar{P}_{u^{\prime} u^{\prime}}$ ) is shown for comparative purposes in Fig. 9-there is no appreciable variation with frequency in $\bar{P}_{u^{\prime} u^{\prime}}$, nor do unsteady measures appear to differ greatly from their counterparts in steady flow under the same mean conditions. The frequency invariance of $\bar{P}_{u^{\prime} u^{\prime}}$ is contrasted by an orderly decrease in amplitude of $\widetilde{P}_{u^{\prime} u^{\prime}}$ with increasing frequency, which may be seen plainly in Fig. 8 despite the scatter in these data. Thus another feature of the dynamic response of boundary-layer turbulence to oscillatory shear is a reduction in amplitude of production of ${ }^{\prime} u^{\prime}$ with increased frequency. The strong similarity between shapes of profiles of $\widetilde{P}_{u^{\prime} u^{\prime}}$ and $\bar{P}_{u^{\prime} u^{\prime}}$, each with coincident peaks at $y^{+} \simeq 9$, indicates that mean and oscillatory production of $u^{\prime} u^{\prime}$ are closely related. Rather than an indcpendent facet of the oscillatory ficld of flow,

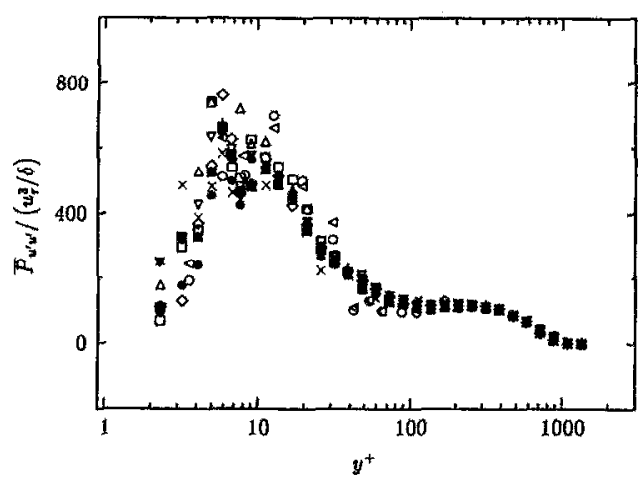

FIG. 9. Boundary-layer profiles of production of $\overline{u^{\prime} u^{\prime}} ; \times, 2.0 \mathrm{~Hz} ; \mathbf{m}, 1.6$ $\mathrm{Hz} ; 0,1.0 \mathrm{~Hz} ; 0,0.8 \mathrm{~Hz} ; \nabla, 0.5 \mathrm{~Hz} ; \square, 0.2 \mathrm{~Hz} ; \Delta, 0.1 \mathrm{~Hz} ; 0$, quasisteady, 4 , steady.

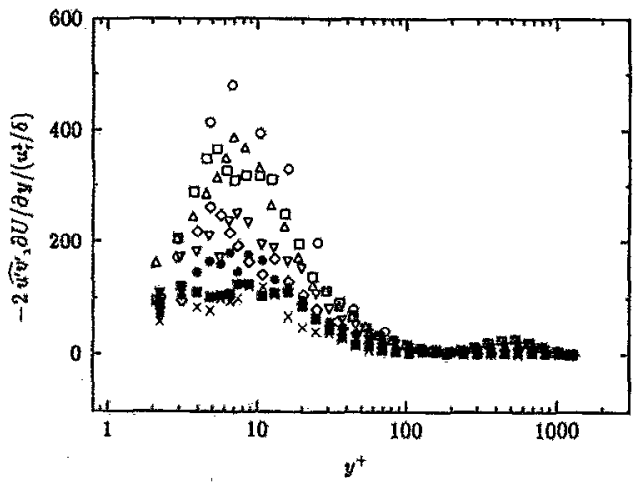

FIG. 10. Boundary-layer profiles of the amplitude of production of $\left\langle u^{\prime} u^{\prime}\right\rangle$ through the action of mean shear upon the oscillatory Reynolds stress; $X$, $2.0 \mathrm{~Hz} ; 1.6 \mathrm{~Hz} ; 1,0 \mathrm{~Hz} ; \diamond, 0.8 \mathrm{~Hz} ; \nabla, 0.5 \mathrm{~Hz} ; \square, 0.2 \mathrm{~Hz} ; \Delta, 0.1 \mathrm{~Hz} ; 0$, quasisteady.

oscillatory production of turbulence appears to be an intense modulation of the local production process of the parent boundary layer.

The amplitudes of each of the major contributors to $\widetilde{P}_{u^{\prime} u^{\prime}}\left(-2 \widetilde{u^{\prime} v^{\prime}} \partial U / \partial y\right.$ and $\left.-2 \overline{u^{\prime} v^{\prime}} \partial \tilde{u} / \partial y\right)$ are shown at their first harmonics in Figs. 10 and 11, respectively. It is clear that both terms make comparable contributions to production of $u^{\prime} u^{\prime}$ at frequencies close to the quasisteady asymptote. This finding is consistent with simple mixing-length turbulence models, which locally relate $\left\langle u^{\prime} v^{\prime}\right\rangle$ to $\partial\langle u\rangle / \partial y$ through a constant of proportionality - the kind of relationship justified on dimensional grounds for turbulent shear flows dominated by a single scale of $v^{\prime}$ and a single turbulent length scale. The growing importance of the additional imposed time scale, brought about by increasing the frequency of shear, manifests itself in a reduction of $-2 \widetilde{u^{\prime} v^{\prime}} \partial U / \partial y$ (and so $\widetilde{P}_{u^{\prime} u^{\prime}}$ ) from its quasisteady level, throughout the boundary layer. Since profiles of $U$ (and so $\partial U / \partial y$ ) have been shown to be invariant with frequency, ${ }^{13}$ this effect results from attenuation of the oscillatory Reynolds-stress amplitude with increasing frequency (or reduced response time). Consequently, extension of turbulence models of this class to unsteady-fiow problems may only be justified close

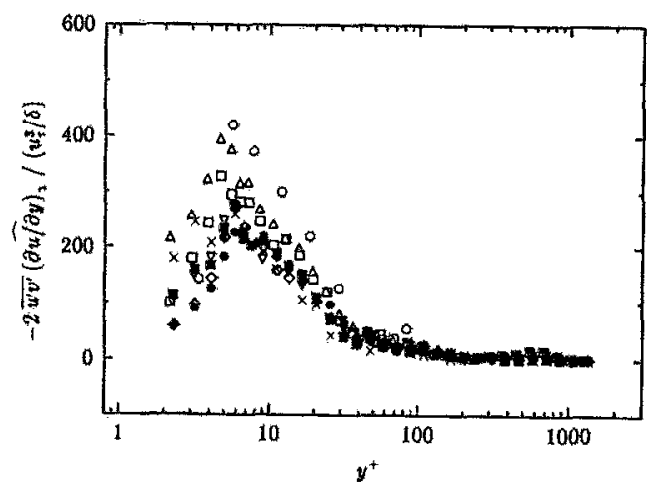

FIG. 11. Boundary-layer profiles of the amplitude of production of $\left\langle u^{\prime} u^{\prime}\right\rangle$ through the action of oscillatory shear upon the mean Reynolds stress; $X$, $2.0 \mathrm{~Hz} ; 1.6 \mathrm{~Hz} ;, 1.0 \mathrm{~Hz} ; 0,0.8 \mathrm{~Hz} ; \nabla, 0.5 \mathrm{~Hz} ; \square, 0.2 \mathrm{~Hz} ; \Delta, 0.1 \mathrm{~Hz} ; 0$, quasisteady. 
to the quasisteady asymptote, unless correction is made for the additional time scale.

The component of $\widetilde{P}_{u^{\prime} u^{\prime}}$ due to $-2 \overline{u^{\prime} v^{\prime}} \partial \tilde{u} / \partial y$ exhibits only a weak sensitivity to frequency variation. Careful inspection of the data of Fig. 11 reveals that this frequency dependence follows the trend in near-wall measures of $\partial \tilde{u} / \partial y$, shown in the inset of Fig. 4 . The indication that $-\overline{u^{\prime} v^{\prime}}$ is scarcely affected by different values for forcing frequency is consistent with the results of several studies. ${ }^{3,13}$ Furthermore, the maxima of both $-2 \overline{u^{\prime} v^{\prime}} \partial \tilde{u} / \partial y$ and $-2 u^{\prime} v^{\prime} \partial U / \partial y$ are at the same position within the boundary layer. This position $\left(y^{+} \simeq 9\right)$ is approximately the one at which production peaks in the underlying mean flow and reinforces the view that oscillatory turbulence production arises as a modulation of the mean production process of the parent boundary layer.

While the information presented in Figs. $8-11$ is believed to be qualitatively accurate, the quantitative nature of the data should be interpreted with caution. These data are the product of a Fourier decomposition of a phase-conditioned measure and a time-averaged measure. One measure is a double correlation of turbulent velocities, deduced from the difference of means of squares, and squares of means; the other is a fitted gradient. The opportunity for systematic errors to accrue during this sequence of data-reduction procedures is therefore large. Conservative estimates of the relative errors in these quantities placed them at about $\pm 20 \%$ of their local values. Consequently, while the trends in these data are believed to be accurate, their absolute values are less trustworthy and may not be appropriate as target data for turbulence modelers.

The two major terms that contributed to production of $\widetilde{v^{\prime} v^{\prime}}\left(-2 \widetilde{v^{\prime} v^{\prime}} \partial V / \partial y\right.$ and $\left.-2 \overline{v^{\prime} v^{\prime}} \partial \tilde{v} / \partial y\right)$ and to production of $-\widetilde{u^{\prime} v^{\prime}\left(v^{\prime} v^{\prime}\right.} \partial U / \partial y$ and $\left.\overline{v^{\prime} v^{\prime}} \partial \tilde{u} / \partial y\right)$ were also measured in this study. Those responsible for generation of $\widetilde{v^{\prime} v^{\prime}}$ were smaller by at least an order of magnitude, as a consequence of the negligibly small values of $V$ and $\tilde{v}$ that were measured-they differed from zero by amounts of the order of the uncertainty in their measurement. The two major terms accounting for production of $-\widetilde{u^{\prime} v^{\prime}}$ were locally nearly half as large as those that generated $\widetilde{u^{\prime} u^{\prime}}$. The general trends in profiles of amplitudes of these terms, the amplitude of their sum, and its size relative to the mean level of production of $-u^{\prime} v^{\prime}$ were very similar to those for $u^{\prime} u^{\prime}$ and are not reported here.

While no measurements of $\widetilde{w^{\prime} w^{\prime}}$ or its production tensor were possible, it is doubtful that there could be any appreciable production in a nominally two-dimensional boundary layer, upon which two-dimensional oscillatory motion was superimposed; the high spanwise uniformity of time-averaged and time-dependent flow features precluded the possibility of any appreciable spanwise gradients. ${ }^{16}$ Thus the production terms for turbulence in this wall-bounded oscillatory shear flow serve a purpose similar to that in a steady pure-shear flow-to concentrate almost the entire production of turbulent kinetic energy in one component of the energy field. In this wall-bounded flow, the $\widetilde{u^{\prime} u^{\prime}}$ component is the prime beneficiary, as a result of modulation of the mean production process in the parent boundary layer.

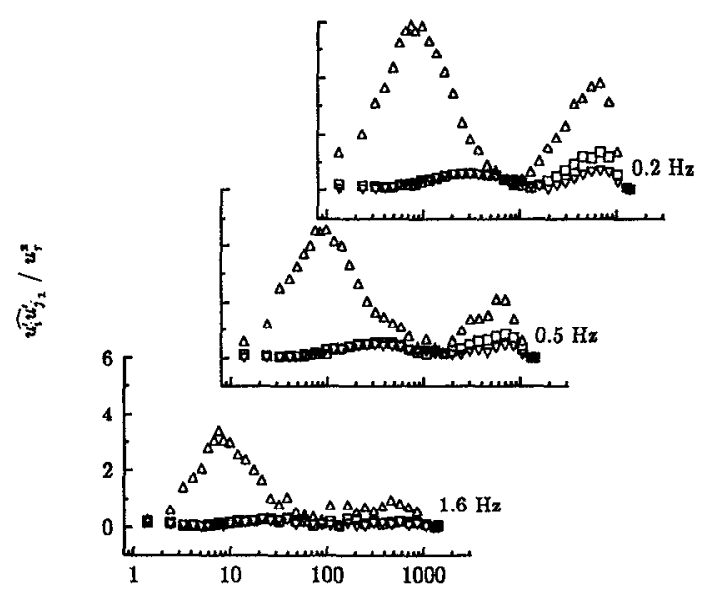

FIG. 12. Boundary-layer profiles of the amplitude of oscillations in components of the Reynolds-stress tensor, normalized in wall units. Amplitudes are plotted at their first harmonics at $0.2,0.5$, and $1.6 \mathrm{~Hz} ; \Delta, \widehat{u^{\prime} u_{1}^{\prime}} / u_{r}^{2} ; \square$, $\widehat{v^{\prime} v_{1}^{\prime}} / u_{r}^{2} ; \nabla,-\widehat{u^{\prime} v_{1}^{\prime}} / u_{r}^{2}$.

\section{RESPONSE OF THE TURBULENCE FIELD}

The oscillatory components of the Reynolds-stress tensor, $\widetilde{u}^{\prime} u^{\prime}, \widetilde{v^{\prime} v^{\prime}}$, and $-\widetilde{u^{\prime} v^{\prime}}$, represent the organized dynamic response of turbulence to imposed oscillatory shear and each was most energetic at its first harmonic, for all frequencies of imposed shear. When these quantities were evaluated as their first-harmonic amplitudes, $\widehat{u^{\prime} u_{1}^{\prime}}$ characteristically accounted for between $60 \%$ and $95 \%$ of the total harmonic content of $\widetilde{u^{\prime} u^{\prime}}$ throughout the boundary layer, at all frequencies of oscillatory shear. The proportional harmonic contents of $\widehat{v^{\prime} v_{1}^{\prime}}$ and $-\widehat{u^{\prime} v_{1}^{\prime}}$ approached these percentages only at the lower frequencies at which this study was conducted. For higher frequencies of oscillatory shear, $\widehat{v^{\prime} v_{1}^{\prime}}$ and $-\widehat{u^{\prime} v_{1}^{\prime}}$ typically contributed around $50 \%$ of the total harmonic content, the remainder being distributed amongst the higher harmonics admitted by the phase-averaging procedure.

Boundary-layer profiles of $\widehat{u^{\prime} u_{1}^{\prime}}, \widehat{v^{\prime} v_{1}^{\prime}}$, and $-\widehat{u^{\prime} v_{1}^{\prime}}$ are shown in Fig. 12, normalized by the square of the mean (and frequency-invariant) friction velocity. These data are plotted for oscillatory shear at three representative frequencies in this study: (i) a low frequency of $0.2 \mathrm{~Hz}$ at which quasisteady flow was approached; (ii) an intermediate frequency of $0.5 \mathrm{~Hz}$; and (iii) a relatively high frequency, $1.6 \mathrm{~Hz}$, for which there was slug-like flow in $\tilde{u}$, described closely by Stokes' solution. Each profile features an inner and an outer peak and the trough separating thesc peaks corresponds to the region over which ensemble-averaged measures and their mean intersect (see Fig. 3). The inner peaks of the $\widehat{u^{\prime} u_{1}^{\prime}}$ profiles are at almost coincident positions within the boundary layer, and again approximately at the one at which peak production takes place in the mean field $\left(y^{+} \simeq 9\right)$, further reinforcing the view that oscillatory turbulence measures arise as a modulation of motions of the underlying mean flow. At any position within the boundary layer, the effect of increasing frequency of oscillatory shear is to cause a mono- 


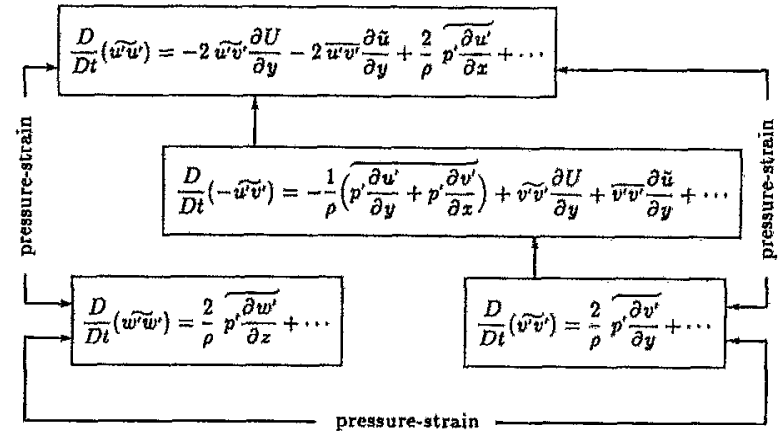

FIG. 13. Coupling of oscillatory Reynoldis-stress transport equations.

tonic decrease in the amplitudes of all three oscillatory turbulence measures, which is demonstrated more convincingly for the full range of frequencies in the detailed account of these data. ${ }^{16}$ For $\widetilde{u^{\prime} u^{\prime}}$ and $-\widetilde{u^{\prime} v^{\prime}}$, this response is consistent with the complementary decrease of $\widetilde{P}_{u^{\prime} u^{\prime}}$ and $\widetilde{P}_{-u^{\prime} u^{\prime}}$ with increased forcing frequency (discussed in Sec. VI). Since measures of $\widetilde{P}_{v^{\prime} y^{\prime}}$ were negligibly small, even considering the reduced magnitude of $\bar{v}^{\prime} v_{1}^{\prime}$ relative to $\widehat{u^{\prime} u_{1}^{\prime}}$, it was inferred that intercomponent transfer must be the principal agent responsible for the detectable magnitudes of $\widehat{v^{\prime} v_{1}^{\prime}}$. It follows that the temporal coupling between oscillatory Reynoldsstress equations and their key production and intercomponent-transfer terms may be represented by the illustration in Fig. 13.

\section{INTERCOMPONENT ENERGY TRANSFER}

Recognizing that the response of the turbulent flow to forced oscillatory shear is predominantly a modulation of motions of the parent boundary layer, one may then examine the extent to which phase relationships between quantities that respond harmonically to forced unsteady turbulent interactions reffect the directions in which turbulent interactions proceed in the parent boundary layer. The data of this study allow phase differences between $\left\langle u^{\prime} u^{\prime}\right\rangle,\left\langle v^{\prime} v^{\prime}\right\rangle$, and

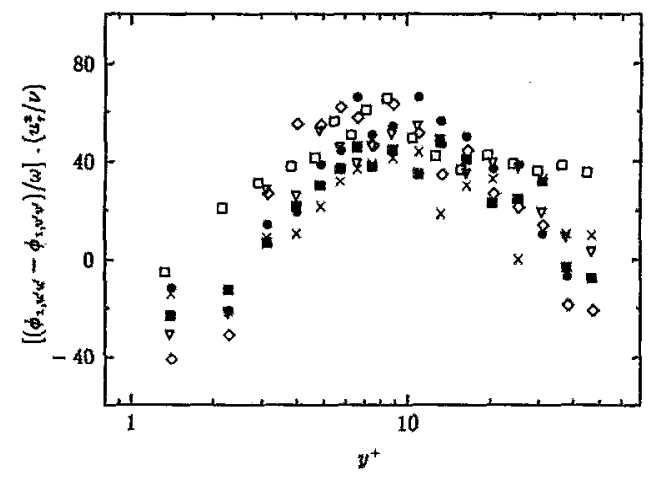

FIG, 14. Near-wall profiles of the normalized time lag between peaks in $\left\langle u^{\prime} u^{\prime}\right\rangle$ and $\left\langle v^{\prime} v^{\prime}\right\rangle ; \square, 0.2 \mathrm{~Hz} ; \nabla, 0.5 \mathrm{~Hz} ; 0,0.8 \mathrm{~Hz} ;, 1.0 \mathrm{~Hz} ; 1.6 \mathrm{~Hz} ; \times$, $2.0 \mathrm{~Hz}$. Note that $\omega$ is the circular frequency of oscillation. $\left\langle-u^{\prime} v^{*}\right\rangle$ to be deduced as cycle-averaged measures, as differences between phases of first harmonics of their Fourier representations. The cycle-averaged differences in phase between $\left\langle u^{\prime} u^{\prime}\right\rangle$ and $\left\langle v^{\prime} v^{\prime}\right\rangle$ are reexpressed as time differences (in viscous units) in Fig. 14, as far from the wall as $y^{+} \simeq 50$. The good general collapse of these data as local frequencyinvariant times scales, in a field of flow characterized by periodic first-harmonic organization of turbulence measures, indicates that these local times scales are derived from the mean flow of the parent boundary layer and are not frequency-dependent facets of the oscillatory component of the flow.

Since intercomponent transfer of turbulent kinetic energy is known to be a strong contributor to the transport equations for $u_{i}^{\prime} u_{j}^{\prime}$ close to the wall, the direction of intercomponent transfer would be associated closely with the sign of phase differences between components of the Reynoldsstress tensor that participate in redistribution of turbulent kinetic energy. In wall-bounded turbulent flow, the redistribution of turbulent kinetic energy between the $u^{\prime} u^{\prime}$ and $v^{\prime} v^{\prime}$ components of the Reynolds-stress tensor is amongst the better understood, and may be examined using the phasedifference data presented in Fig. 14. From these data, it is clear that $\left\langle v^{\prime} v^{\prime}\right\rangle$ leads $\left\langle u^{\prime} u^{\prime}\right\rangle$ between the measurement position closest to the wall and $y^{+} \simeq 2.5$, whereas $\left\langle u^{\prime} u^{\prime}\right\rangle$ leads $\left\langle v^{\prime} v^{\prime}\right\rangle$ beyond this point. The lead of $\left\langle u^{\prime} u^{\prime}\right\rangle$ over $\left\langle v^{\prime} v^{\prime}\right\rangle$ peaks at $y^{+} \simeq 9$ before receding toward zero at $y^{+} \simeq 40$. The corresponding phase relationships between $\left\langle u^{\prime} u^{\prime}\right\rangle$ and $\left\langle-u^{\prime} v^{\prime}\right\rangle$ and $\left\langle v^{\prime} v^{\prime}\right\rangle$ and $\left\langle-u^{\prime} v^{\prime}\right\rangle$ were equally well organized.

The physical interpretation placed on the phase relationships between $\left\langle u^{\prime} u^{\prime}\right\rangle$ and $\left\langle v^{\prime} v^{\prime}\right\rangle$ is that, within the first few viscous units beyond the wall, $\left\langle u^{\prime} u^{\prime}\right\rangle$ leads $\left\langle u^{\prime} u^{\prime}\right\rangle$ because the direction of redistribution of turbulent kinetic energy is from $\left\langle v^{\prime} v^{\prime}\right\rangle$ to $\left\langle u^{\prime} u^{\prime}\right\rangle$ (and $\left.\left\langle w^{\prime} w^{\prime}\right\rangle\right)$. Since production of turbulence is relatively small in this particular region, intercomponent transfer would play a major role in the budgets for $\left\langle u^{\prime} u^{\prime}\right\rangle$ and $\left\langle v^{\prime} v^{\prime}\right\rangle$ [Eq. (1)], and so a time lead of peak levels of $\left\langle v^{\prime} v^{\prime}\right\rangle$ relative to those of $\left\langle u^{\prime} u^{\prime}\right\rangle$ indicates redistribution from $\left\langle u^{\prime} u^{\prime}\right\rangle$ to $\left\langle u^{\prime} u^{\prime}\right\rangle$. Moreover, these time scales are derived from the underlying mean flow and so this effect appears to be an experimental verification of the wall impingement or splatting effect of redistribution of $\overline{v^{\prime} v^{\prime}}$ to $\overline{u^{\prime} u^{\prime}}$ and $\overline{w^{\prime} w^{\prime}}$, identified by Moin and $\operatorname{Kim}^{17}$ in their numerical investigation of turbulent channel flow.

The phase relation between $\left\langle u^{\prime} u^{\prime}\right\rangle$ and $\left\langle u^{\prime} v^{\prime}\right\rangle$ reverses its sign at $y^{+} \simeq 2.5$. This location is very close to the one $\left(y^{+} \simeq 4\right)$ at which the component of the pressure-strain tensor in the $\overline{u^{\prime} u^{\prime}}$ transport equation changes its role from enhancing the level of $\overline{u^{\prime} u^{\prime}}\left(y^{+} \leqslant 4\right)$ to reducing it $\left(y^{+} \approx 4\right)$, according to the results of Moin and Kim. ${ }^{17}$ This general agreement appears to be remarkably good since diffusive and dissipative effects play additional roles in determining the phase relation between $\left\langle u^{\prime} u^{\prime}\right\rangle$ and $\left\langle v^{\prime} v^{\prime}\right\rangle$, obscuring the clarity with which the difference in phase between $\left\langle u^{\prime} u^{\prime}\right\rangle$ and $\left\langle v^{\prime} v^{\prime}\right\rangle$ represents the direction of redistribution of turbulent kinetic energy between these components. The differing flow geometries and Reynolds numbers ( $\mathrm{Re}=860$ in this experiment as opposed to 640 in the channel-flow simulation, ${ }^{17}$ 
when Reynolds number is based upon the mean friction velocity $u_{r}$ ) may also explain the small discrepancy observed.

The magnitude of the time lead/lag between $\left\langle u^{\prime} u^{\prime}\right\rangle$ and $\left\langle v^{\prime} v^{\prime}\right\rangle$ is indicative of the coupled time scales for redistributive motions and the subsequent evolutions of the coupled system of Reynolds-stress transport equations. As such, it is not a clear indicator of whether the mode of redistribution is predominantly "rapid," "slow," or whether both modes are important in this flow. However, the greatest time leads of $\left\langle u^{\prime} u^{\prime}\right\rangle$ over $\left\langle v^{\prime} v^{\prime}\right\rangle$ are at $y^{+} \simeq 9$, coincident with the position of peak production of $u^{\prime} u^{\prime}$ in the boundary layer. Since production of $u^{\prime} u^{\prime}$ dominates the turbulence production tensor close to the wall in turbulent boundary layers, the coincidence of these peak levels is indicative of a strong association between the time scales of agents of redistribution of turbulent kinetic energy and the anisotropy of the turbulence production tensor, consistent with the form of well-known turbulence models for time-averaged components of the intercomponent-transfer terms in Eq. (1). ${ }^{18,19}$

The time scales of energy redistribution shown in Fig. 14 are also of the same order as other characteristic time scales of the parent boundary layer. Close to the wall, estimates of $k / \epsilon$ were typically around 40 viscous units, and $\delta / U_{\infty}$, the turnover time of a large eddy of the mean flow, corresponded to 35 viscous units in time. The general comparability of these time scales with the data of Fig. 14 reinforces the contention that it is the turbulent motions of the underlying mean flow that determine the time delay between peaks levels in components of $\left\langle u_{i}^{\prime} u_{j}^{\prime}\right\rangle$ when oscillatory shear is superimposed upon the boundary layer. One may therefore interpret the superposition of oscillatory motion upon boundary-layer turbulence as a forced perturbation of the robust turbulent motions that dominate the underlying flow, whose response reveals their temporal characteristics. The time differences in response of turbulence measures, necessitated by oscillatory shear, are controlled by motions of the mean flow that are so resilient to periodic deformation that the same local time scale of response is found, regardless of the forcing frequency. Thus study of the response to external forcing may be viewed as a diagnostic technique for probing local temporal features of the turbulent flow, similar to that used for study of linear dynamical systems with few degrees of freedom.

Some closing comments are in order concerning the results presented in this section. Phase-difference data are presented for all frequencies of this study except $0.1 \mathrm{~Hz}$. These data could not be considered reliable, since the combination of very small phases and phase differences, scaled by large oscillation periods, resulted in high levels of uncertainty. Measurements of phase difference were not presented beyond $y^{+}=50$ at any frequency, since their uncertainty again became excessively large. While the number of recorded ensembles appeared to provide adequate descriptions of many statistical measures of turbulent activity, profiles of the phase of $\widetilde{u_{i}^{\prime} u_{j}^{\prime}}$ exhibited excessive scatter beyond the nearwall region of the boundary layer, where the magnitude of the forcing shear was negligible. The increased scatter was attributed to the greater importance of diffusive effects in this region, rendering turbulent motions less coherent and thereby requiring averaging over greater numbers of ensembles to assure adequate convergence in sensitive statistical measures such as Fourier phases.

\section{CONCLUSIONS}

The results of this study provide a basis for broader insights into the temporal behavior of boundary-layer turbulence while undergoing a well-defined form of superposed unsteady deformation-oscillatory shear. Over a broad range of frequencies, the dynamic response of the turbulence field to forced excitation is primarily a first-harmonic organization in magnitude of components of the Reynolds-stress tensor. Temporal production of turbulence is characterized as an intense modulation of turbulence-producing motions of the parent boundary layer, which are resilient to periodic deformation.

Close to the wall, time-dependent redistribution of turbulent kinetic energy amongst its components is driven by motions of the underlying mean flow, with only a weak dependence on oscillatory motions. The phase relationships between these components, in response to forced oscillatory shear, act as a diagnostic tool for the parent boundary layer, indicating the local direction and time scale for turbulent kinetic energy redistribution. They provide verification of the wall-impingement effect, previously identified only in numerical simulation of steady turbulent wall-bounded flow.

\section{ACKNOWLEDGMENTS}

The experimental program on which this work is based was carried out at Stanford University under the support of the U.S. Army Research Office under Contract No. DAAG 29-83-K-0056. Much of the subsequent analysis was carried out under the support of The University of Michigan. These sources of support are gratefully acknowledged.

'S. J. Kline, B. J. Cantwell, and G. M. Lilly, in Proceedings of the $1980-81$ AFOSR-HTTM-Stanford Conference on Complex Turbulent Flows (Department of Mechanical Engineering, Stanford University, 1982), Vol. 1.

${ }^{2}$ S. K. F. Karlsson, J. Fluid Mech. 5, 622 (1959).

${ }^{3}$ L. Shemer, I. Wygnanski, and E. Kit, J. Fluid Mech. 153, 313 (1985).

${ }^{4}$ A. A. Schachenmann and D. O. Rockwell, J. Fluids Eng. Trans. Am. Soc. Mech. Eng. 98, 695 (1976).

${ }^{5} \mathrm{G}$. Binder and J. L. Kueny, in Proceedings of the 3rd Symposium on Turbulent Shear Flows, The University of California, Davis, CA, 1981.

'S. W. Tu and B. R. Ramaprian, J. Fluid. Mech. 137, 31 (1983).

${ }^{7}$ R. L. Simpson, B. G. Shivaprasad, and Y. T. Chew, J. Fluid Mech. 127, 219 (1983).

${ }^{8}$ M. Acharya and W. C. Reynolds, Report No. TF-8, Department of Mechanical Engineering, Stanford University, 1975.

${ }^{9}$ W. Kebede, B. E. Launder, and B. A. Younis, Proceedings of the 5th Symposium on Turbulent Shear Flows, Cornell University, Ithaca, NY, 1985.

${ }^{10} \mathrm{P}$. Blondeaux and M. Colombini, in Ref. 9.

"T. Cebeci, Proc. R. Soc. London Ser. A 335, 225 (1977).

${ }^{12}$ D. C. Wilcox, AIAA J. 26, 11 (1988).

${ }^{13}$ G. J. Brereton, W. C. Reynolds, and R. Jayaraman, J. Fluid Mech. 221, 131 (1990).

${ }^{14}$ A. K. M. F. Hussain and W. C. Reynolds, J. Fluid Mech. 41, 241 (1970).

${ }^{15}$ S. L. Marple, Digital Spectral Analysis with Applications (Prentice-Hall, Englewood Cliffs, NJ, 1987).

${ }^{16} \mathrm{G}$. J. Brereton and W. C. Reynolds, Report No. TF-29, Department of Mechanical Engineering, Stanford University, 1987.

${ }^{17}$ P. Moin and J. Kim, J. Fluid Mech. 118, 341 (1982).

${ }^{18}$ J. C. Rotta, Turbulente Strömungen (Teubner, Stuttgart, 1972),

${ }^{19}$ D. Naot, A. Shavit, and M. Wolfshtein, Isr. J. Technol, 8, 259 (1970). 